\title{
The Explaining of "Magic" Nuclear Numbers by a Quasi-Crystalline Nuclear Model, of Possible Cold Genesis
}

\author{
Marius Arghirescu \\ State Office for Inventions and Trademarks, OSIM, Romania \\ Email: arghirescu.marius@osim.ro
}

\begin{abstract}
The paper is based on a cold genesis theory of the author, (CGT), in which the proton results as formed by a neutral $\mathrm{N}^{\mathrm{p}}$ cluster of degenerate electrons and an attached positron with degenerate spin and magnetic moment. Also, the neutron results in a "dynamide" model, as formed by a proton and a degenerate electron with degenerate spin and magnetic moment, with its centroid incorporated in the proton quantum volume and rotated around the proton center.

In the paper it is shown that the stable nuclei with "magic" or semi-"magic" number of protons or and neutrons: $2 ; 8 ; 20 ; 28 ;(32,36,40) ; 50 ; 82 ; 126$, are retrieved by a quasi-crystalline nuclear model of ground state $\mathrm{T} \rightarrow 0 \mathrm{~K}$, as sum of quasi-crystalline forms with integer number of alpha particles with $2 \mathrm{n}^{2}$ protons and $4 \mathrm{n}^{2}$ nucleons having small deformation parameter, for the double 'magic' nuclei. This possibility may be explained by the dynamide model of neutron of CGT by the hypothesis of $\alpha^{0}$ neutron clusters cold forming at $\mathrm{T} \rightarrow 0 \mathrm{~K}$ and the generating of small square forms of neutral $\alpha^{0}$ particles which are transformed into nuclei by $\alpha^{0}$-particles transforming into $\alpha^{+}$and $\alpha^{2+}$ particles which attract new $\alpha^{0}$-particles, the process being repeated until the forming of double magic nuclei which may attract nucleons or $\alpha^{0}$-clusters transformed thereafter into $\alpha^{+}$clusters, or of nuclei with "magic" mass number. According to the model, the nucleus ${ }^{208} \mathrm{~Pb}_{82}$ corresponds to the initial form: ${ }^{208} \mathrm{~N}_{104}\left(\mathrm{Z}=2\left(4^{2}+6^{2}\right)\right)$ in which 22 attracted $\alpha^{0}$-clusters were transformed into $\alpha^{+}$clusters, by $\beta$ radiation emission

The proposed model predicts that the nuclei with $\mathrm{A}=4\left(5^{2}+7^{2}\right)=296, \mathrm{~A}=4 \times\left(6^{2}+2 \times 4^{2}+2 \times 2^{2}=304\right)$ nucleons and $Z=114 \div 120$ are more stable in the ground state than the forms: $114 / 184,120 / 182$ predicted with the "nuclear shells" model. Also, it results as possible the forming of cold semi"magic" nuclei with hexagonal symmetry, with the mass number $A=\Sigma\left(3 \times 4 n^{2}\right),(n=1 \ldots 5)$.
\end{abstract}

Keywords: crystalline nuclear model, magic nuclei, dynamide neutron model, tetra-neutron, cold genesis

\section{Introduction}

- In a Cold Genesis Theory of Matter and Fields of the author, (CGT-[1-4]), based on the Galilean relativity [6], the discovered elementary particles are explained by a vortexial model, of composite fermion type, as Bose -Einstein Condensate of $\mathrm{N}^{\mathrm{p}}$ gammons considered as thermalized pairs: $\gamma^{*}=\left(\mathrm{e}^{-} \mathrm{e}^{+}\right)$ of axially coupled electrons with opposed charges which became degenerate electrons inside the neutral $\mathrm{N}^{p}$ cluster, i.e. - quasi-electrons with diminished mass, charge and magnetic moment: $\mathrm{m}_{\mathrm{e}}^{*} \approx 0.81 \mathrm{~m}_{\mathrm{e}}$; $\mathrm{e}^{*} \approx(2 / 3) \mathrm{e} ; \mu_{\mathrm{e}}^{*} \approx \mu_{\mathrm{e}}\left(2.79 \mathrm{~m}_{\mathrm{e}} / \mathrm{m}_{\mathrm{P}}\right) \approx \mu_{\mathrm{P}},[4,5]$.

The motivation of the theory results from the fact that neither the Big-Bang theory nor the quantum vacuum fluctuations theory not explain physically the generation of the specific properties of the elementary particles (mass spectrum, spin, magnetic moment, electric charge, etc.) and of the fundamental fields, even if the Standard Model of particles, the Quantum chromodynamics and the Electro-weak theory are compatible with a lot of observational data.

- The theory uses an electron model with the charge $e=S^{0} / k_{1}$ contained by its surface $S^{0}=4 \pi \mathrm{a}^{2}$ of radius: $\mathrm{a}=1.41 \mathrm{fm}$, (close to the value of the nucleon radius resulted from the expression of the nuclear volume: $\mathrm{r}_{\mathrm{r}} \approx 1.25 \div 1.5 \mathrm{fm}$ ) and with an exponential variation of its density and of quanta density variation inside the electron's quantum volume:

$$
\begin{gathered}
\rho_{e}=\rho_{e}^{0} \cdot e^{-r / \eta} ;\left(\rho_{e}^{0}=22.24 \mathrm{~kg} / \mathrm{m}^{3} ; \eta=0.965 \mathrm{fm}\right) ; \\
\rho_{e}(a)=\mu_{0} / k_{1}^{2}=5.17 \times 10^{-13} \mathrm{~kg} ;\left(k_{1}=4 \pi \cdot a^{2} / e\right)
\end{gathered}
$$


- The particle's magnetic moment $\mu_{\mathrm{e}}^{*}$ results in CGT as etherono-quantonic vortex: $\Gamma_{\mu}^{*}(\mathrm{r})=\Gamma_{\mathrm{A}}+\Gamma_{\mathrm{B}}$, of heavy ("sinergonic") etherons $\left(\mathrm{m}_{\mathrm{s}} \approx 10^{-60} \mathrm{~kg}\right)$ - generating the magnetic potential A and of quantons $\left(\mathrm{m}_{\mathrm{h}}=\mathrm{h} \cdot 1 / \mathrm{c}^{2}=7.37 \times 10^{-51} \mathrm{~kg}\right)$ - generating vortex-tubes $\xi_{\mathrm{B}}$ that materializes the $\mathbf{B}$-field lines of the magnetic induction.

- The virtual radius: $\mathrm{r}_{\mu}{ }^{\mathrm{n}}$ of the proton's magnetic moment, $\mu_{\mathrm{p}}$, results by a degenerate Compton radius of an attached positron, which decreases when the protonic positron is included in the neutral $\mathrm{N}^{\mathrm{p}}$ cluster volume, from the value: $r_{\mu}{ }^{e}=3.86 \times 10^{-13} \mathrm{~m}$, to the value: $r_{i}=r_{\mu}{ }^{p}=0.59 \mathrm{fm}$, as a consequence of the increasing of the impenetrable quantum volume mean density in which is included the protonic positron's centroid, $\mathrm{m}_{0}$, from the value: $\bar{\rho}_{e}$ to the value: $\bar{\rho}_{n} \cong f_{d} \cdot N^{p} \cdot \bar{\rho}_{e}$, in which: $\mathrm{k}_{\mathrm{P}}=\bar{\rho}_{n} / \bar{\rho}_{e}-$ the gyromagnetic ratio; $\bar{\rho}_{e} ; \bar{\rho}_{n}$ - the mean density of electron and of nucleon; $f_{d}$-the degeneration coefficient which gives the quasielectron mass $\mathrm{m}_{\mathrm{e}}{ }^{*} \approx 0.81 \mathrm{~m}_{\mathrm{e}}$.

The neutral $\mathrm{N}^{\mathrm{p}}$-cluster of nucleons and of other astro-particles may be considered as composed by three cold formed quarks with preonic structure based on a neutral preon $z^{0} \approx 34 \mathrm{~m}_{\mathrm{e}},[1-4]$.

- The superposition of the $\left(\mathrm{N}^{\mathrm{p}}+1\right)$ quantonic vortices: $\Gamma_{\mu}{ }^{*}$ of the proton' quasielectrons generates inside a volume with the radius: $\mathrm{r}_{\mu}{ }^{a}=2.35 \mathrm{fm}[1-4]$ a total dynamic pressure: $P_{n}=(1 / 2) \rho_{n}(r) \cdot c^{2}$ which gives a nuclear potential: $\mathrm{V}_{\mathrm{n}}(\mathrm{r})$, in an Eulerian form, having a variation according to eqn.:

$$
V_{n}(r)=v_{i} P_{n}(r)=V_{n}^{0} \cdot e^{-r / \eta^{*}} ; \quad V_{n}^{0}=v_{i} P_{n}{ }^{0}=\left(v_{i} / 2\right) \rho_{n}{ }^{0} \cdot c^{2} \quad \text { with: } \eta^{*}=0.8 \mathrm{fm}[2],
$$

$\left(v_{i}\left(r_{i}\right) \approx 0.86 \div 0.9 \mathrm{fm}^{3}\right.$ - the nucleon's impenetrable quantum volume of nuclear interaction).

- Also, the neutron results in CGT by a specific "dynamide" model, with a degenerate electron with degenerate magnetic moment: $\mu_{\mathrm{e}}^{\mathrm{s}} \approx-4.6 \mu_{\mathrm{N}}\left(\mu_{\mathrm{N}}-\right.$ the nuclear magneton) rotated inside the quantum volume of a proton by the etherono-quantonic vortex $\Gamma_{\mathrm{P}}$ of the protonic magnetic moment $\mu_{\mathrm{p}}$, with a speed $\mathrm{v}_{\mathrm{e}} \approx 1.7 \times 10^{-2} \mathrm{c}$, to an orbital with a radius: $\mathrm{r}_{\mathrm{e}}{ }^{*} \approx 1.283 \mathrm{fm}$, under dynamic equilibrium of forces on tangent and radial directions, [3-5].

There are also some relative recent experiments which are in concordance with CGT, such as: i) the possibility to "split" the gamma- quantum into a pair e+ - e- with an electrostatic energy

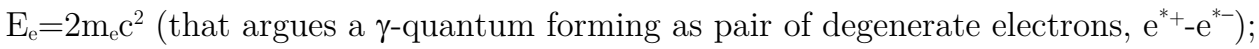

ii) the experimental obtaining of a BEC of photons, (a "super-photon"), by a German team (2010), (proving the existence of photonic rest mass);

iii) the experimental evidencing of a $34 \mathrm{~m}_{\mathrm{e}}$ neutral boson, (preon of cold genesis - in CGT), by a Hungarian team, (but considered as quantum of a fifth force, of leptons to quarks binding, [6]);

iv) the almost same size order of the radius of scattering centers determined inside the electron and inside the nucleon, ( $10^{-18} \mathrm{~m}$ [7]- value considered also for quarks [8], but being the radius of a superdense electronic kernel (centroid), in CGT, [1-5]).

It is known also the oppinion that the majority of the atomic elements are synthesized inside the stars, at very high temperature, for example, in a supernova explosion, in conditions in which the kinetic energy of the lightest nuclei $(\mathrm{H}, \mathrm{D}, \mathrm{He})$ exceeds the repulsive coulombian potential and favores their fusion.

A question that arises is that at least the "magic" nuclei may be formed also "at cold" ( $\mathrm{T} \rightarrow 0 \mathrm{~K})$, as quasi-crystalline clusters of protons and neutrons.

\section{The Explanation of the Nuclear "Magic" Numbers of Nuclear Stability through a Quasi-Crystalline Nuclear Model}

By the solitonic "dynamide" model of neutron reconsidered in CGT [1-4] and in concordance with the observations that shown a maximum nuclear stability for even-even nuclei, the nuclear model resulted from CGT for the ground state $\mathrm{T} \rightarrow 0 \mathrm{~K}$ is of quasi-crystalline type, corresponding to the extremeuniparticle model (of Schmidt type), to the "nuclear molecule" model, to the alpha particle cluster model and to the rotating rigid vibratory core model.

According to this quasi-crystalline model, at $\mathrm{T} \rightarrow 0 \mathrm{~K}$ the nucleus consists of symmetrical overlapping of square forms with an integer number of alpha particles, the unpaired nucleon(s) being rotated around this quasi-crystalline nucleus by the quantum vortex of the nuclear magnetic moment, which explains according to the theory, the centrifugal nuclear potential, or being attached to the double 'magic' central part as $\alpha^{0}$ clusters of four neutrons. 
The stable nuclei with "magic" number of protons or and neutrons (the most known being: 2; 8; 20; 28; $40 ; 50 ; 82 ; 126)$ are found- according to the model, as symmetrical quasi-crystalline forms resulted from overlapping of integer number of alpha particles, with $A=\Sigma\left(4 n^{2}\right)$ nucleons $(n=1,2, \ldots 7)$ and $Z=\Sigma\left(2 n^{2}\right)$ protons- for the double magic nuclei, with relative small deformation parameter $\delta$, (figure 1), with a total number $\mathrm{K}_{\mathrm{a}}=(1 ; 2 ; 3 ; 4),\left(\mathrm{K}_{\mathrm{b}}, \mathrm{K}_{\mathrm{c}}\right)=(0 ; 1 ; 2)$ of squared or hexagonal forms with $\mathrm{n}_{\mathrm{a}}^{2}, \mathrm{n}_{\mathrm{b}}{ }^{2}, \mathrm{n}_{\mathrm{c}}^{2},\left(3 \mathrm{n}_{\mathrm{a}}^{2}\right.$, $\left.3 \mathrm{n}_{\mathrm{b}}^{2}, 3 \mathrm{n}_{\mathrm{c}}^{2}\right) \alpha$-particles on the levels a., b., c.:

Double 'magic' forms: $(\mathrm{Z}=\mathrm{A} / 2): \mathrm{A}=4^{1},\left({ }^{4} \mathrm{He}_{2}\right) ; \mathrm{A}=4 \times 2^{2}=16,\left({ }^{16} \mathrm{O}_{8}\right) ; \mathrm{A}=4 \times\left(3^{2}+1^{2}\right)=40,\left({ }^{40} \mathrm{Ca}\right)$; $\mathrm{Z}=2 \times\left(4^{2}+5^{2}\right)=82, \mathrm{~A}=4 \times\left(4^{2}+6^{2}\right),\left({ }^{208} \mathrm{~Pb}_{82}\right.$-double 'magic' $)$.

Simple 'magic' or 'semi-magic' nuclear forms: $A=4 \times\left(3^{2}+2 \times 1^{2}\right),\left({ }^{44} \mathrm{Ca}\right.$ - stable isotope);

$\mathrm{A}=4 \times 4 \times 2^{2},\left({ }^{64} \mathrm{Zn}\right.$-the most stable/abundant isotope $)$

$\mathrm{A}=4 \times\left(3^{2}+2^{2}\right),\left({ }^{52} \mathrm{Cr}\right.$ - stable/abundant isotope $) ; \mathrm{A}=4 \times\left(3^{2}+2 \times 2^{2}\right)=68,\left({ }^{68} \mathrm{Zn}\right.$-stable/abundant isotope);

$\mathrm{A}=4 \times\left(3^{2}+2^{2}+1^{2}\right)=56$, or $\mathrm{A}=4 \times\left(3 \times 2^{2}+2 \times 1^{2}\right)=56 ;\left({ }^{56} \mathrm{Fe}\right.$ - the most stable/abundant is. $)$;

$\mathrm{A}=4 \times 2 \times 3^{2}=72,\left({ }^{72} \mathrm{Ge}-\right.$ stable/ab. is. $) ; \mathrm{A}=4 \times\left(2 \times 3^{2}+2 \times 1^{2}\right)=80,\left({ }^{80} \mathrm{Kr} ;{ }^{80} \mathrm{Se}-\right.$ stable/ab. is. $)$;

$\mathrm{A}=4 \times\left(2 \times 3^{2}+2^{2}+1^{2}\right)=92$, ( ${ }^{92} \mathrm{Zr}-$ stable is. $) ; \mathrm{A}=4 \times\left(4^{2}+2 \times 2^{2}\right)=96,\left({ }^{96} \mathrm{Mo}-\right.$ stable/ab. is. $)$;

$\mathrm{A}=4 \times 3 \times 3^{2}=108$, ( ${ }^{108} \mathrm{Pd}$ - stable/ab.is. $) ; \mathrm{A}=4 \times\left(5^{2}+2^{2}\right)=116,\left({ }^{116} \mathrm{Sn}\left(\mathrm{Z}=2 \cdot 5^{2}=50\right)-\right.$ stable/ab. is. $)$;

$\mathrm{A}=4 \times 2 \times 4^{2},\left({ }^{128} \mathrm{Xe}-\right.$ stable is. $) ; \mathrm{A}=4 \times\left(4 \times 3^{2}+2 \times 1^{2}\right)=152,\left({ }^{152} \mathrm{Sm},(\mathrm{Z}=62)\right.$-the most ab. is. $)$;

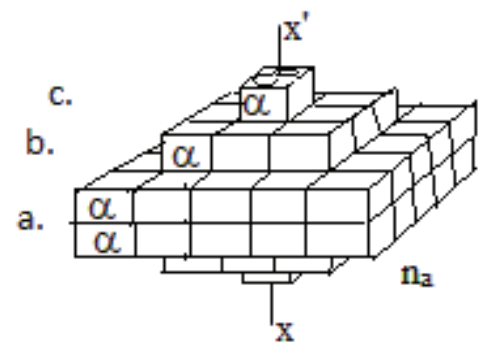

Figure 1. "Magic" form of a quasi-crystal nucleus

For Ge, Zr, Kr, Sn, etc., the greater number of neutrons $(\mathrm{N}>\mathrm{Z})$ may be explained in the model by the attraction of some neutron $\alpha^{0}$-clusters or $1 \div 3$ excess neutrons in the nuclear field of a double magic core, at $\mathrm{T} \rightarrow 0$, these neutrons or $\alpha^{0}$-clusters remaining un-transformed or generating a nuclear quasi-crystalline form with "magic" mass number, $\mathrm{A}=\Sigma\left(4 \mathrm{n}^{2}\right)$, in which some neutral $\alpha^{0}$-clusters are only partially transformed into $\alpha^{1+}$-clusters.

For example, ${ }^{116} \mathrm{Sn}$ may be in a quasi-crystalline form: $\mathrm{A}=4 \times 5^{2}+4 \times 4$, i.e.- a double magic form with $\mathrm{Z}=2 \times 5^{2}$ and four symmetrically attached $\alpha^{0}$-clusters. Also, ${ }^{80} \mathrm{Kr}$ (stable isotope, $\mathrm{A}=4 \times\left(2 \times 3^{2}+2 \times 1^{2}\right)=80$ ), may be considered as formed by a double 'magic' quasi-crystalline form with $\mathrm{Z}=2 \times 2 \times 3^{2}$ and two symmetrically attached $\alpha^{0}$-clusters.

The fact that the nuclei such as ${ }^{40} \mathrm{Ca},{ }^{52} \mathrm{Cr},{ }^{56} \mathrm{Fe},{ }^{72} \mathrm{Ge},{ }^{80} \mathrm{Se},{ }^{152} \mathrm{Sm},{ }^{108} \mathrm{Pd}$, are the most abundant isotopes is explained by the conclusion that it results as quasi-crystalline forms in the ground state.

The fact that the stable isotope ${ }^{90} \mathrm{Zr}_{40}$ (with 'magic' $\mathrm{Z}$ and $\mathrm{N}$ ) is the most abundant is explained by the conclusion that it resulted from the quasi-crystalline form ${ }^{92} \mathrm{Zr}$ ( $\mathrm{N}=52$-also stable/abundant isotope) by the losing of two corner neutrons at higher nuclear temperature, being explained the fact that $\mathrm{N}=50$ was found also as 'magic' number of neutrons [9], in this case.

The "magic" form of the nucleus ${ }^{208} \mathrm{~Pb}_{82}$ corresponds -according to the model, to an initial double "magic" quasi-crystalline form: ${ }^{208} \mathrm{~N}_{104}, \quad\left(\mathrm{~A}=4 \times\left(4^{2}+6^{2}\right)\right)$ in which 22 protons were transformed into neutrons with beta emission, giving $\mathrm{Z}=82$.

Similarly can be formed a nucleus with $A=4\left(5^{2}+7^{2}\right)=296$ nucleons, with $Z=114 \div 120$, (close to the predicted (quasi)stable form: 114/298 predicted with the "nuclear shells" model, [9]). The nucleus with $\mathrm{A}=296(\mathrm{Z}=119 ; 120)$ was experimentally obtained by the reactions:

$$
50 \mathrm{Ti}+249 \mathrm{Bk} \rightarrow 296(119)+3 \mathrm{n} ; \quad 50 \mathrm{Ti}+249 \mathrm{Cf} \rightarrow 296(120)+3 \mathrm{n}
$$

Other 'magic' nuclei may be explained by the model in a similar way:

- The double magic nucleus ${ }^{48} \mathrm{Ca}$ may be considered as composed by 3 square forms with $2^{2}=4 \quad \alpha$ particles:

$$
\mathrm{A}=4 \times\left(3 \times 2^{2}\right)=48, \quad \text { in which } 4 \text { protons were transformed into neutrons; }
$$


- The double magic nucleus ${ }^{56} \mathrm{Ni}$ may be considered as composed by 3 square forms with $2^{2}=4 \alpha$-particles and one $\alpha$-particle on each side: $A=4 \times\left(3 \times 2^{2}+2 \times 1^{2}\right)=56$;

- The double magic nucleus ${ }^{96} \mathrm{Zr}_{40}$ may be derived from quasi-crystalline square forms of 24 alpha particles:

$$
\mathrm{A}=4 \times\left(4^{2}+2 \times 2^{2}\right)=96
$$

in which 8 protons were transformed into neutrons by electrons "capturing" or by $\beta^{+}$disintegration.

Other stable nuclei which may be explained by the proposed quasi-crystalline model are:

- The nucleus with $\mathrm{A}=4 \times\left(2 \times 4^{2}+2 \times 2^{2}\right)=160$; (Dysprosium: ${ }^{160}$ Dy -stable isotope);

- The nucleus with $\mathrm{A}=4 \times\left(5^{2}+2 \times 3^{2}\right)=172 ; \quad$ (Ytrium: ${ }^{172} \mathrm{Yt}$ - stable isotope);

- The nucleus with $\mathrm{A}=4 \times\left(5^{2}+2 \times 3^{2}+2 \times 1^{2}\right)=180 ; \quad\left({ }^{180} \mathrm{Hf}(\mathrm{Z}=72)\right.$-stable isotope $)$;

Similarly may result as more stable than the adjacent isotopes, the nuclear forms:

- $\mathrm{A}=272=4 \times\left(6^{2}+2 \times 4^{2}\right)=4 \times\left(2 \times 5^{2}+2 \times 3^{2}\right)$ : ${ }^{272} \mathrm{Bh}$ (Bohrium, $\mathrm{t}_{1 / 2}=11$ s -close to Hassium 270 , considered double magic in the form: $\mathrm{Z}=108 ; \mathrm{N}=162)$;

- $A=4 \times\left(2 \times 5^{2}+2 \times 3^{2}+2 \times 1^{2}\right)=280$; (Rg-Roentgenium, which decays with $\alpha$-particles emission $)$.

Based on the calculation of pairing gap, two neutron separation energy and the shell correction energy, M. Bhuyan, S. Patra and Ahmad found Z $=120$ as the next proton magic number and N=172, 182/184, 208 and 258 as the subsequent neutron magic numbers, [10].

Also, Biswal, Bhuyan et al.,[11], based on a newly developed approach, of simple effective interaction, found that the combination of magic nucleus occurs at $\mathrm{N}=182(\mathrm{Z}=114,120,126)$, predicting a long halflife for ${ }^{292} 120$ and ${ }^{304} 120$, [12].

According to the quasi-crystalline model, it result as 'magic' at $\mathrm{T} \rightarrow 0$ the nuclei with $\mathrm{A}=304$, corresponding to $\mathrm{Z}=120, \mathrm{~N}=184$ and with $\mathrm{A}=296$, corresponding to $\mathrm{Z}=114, \mathrm{~N}=182$, in the quasicrystalline forms:

$$
\mathrm{A}=4 \times\left(6^{2}+2 \times 4^{2}+2 \times 2^{2}\right)=304(\mathrm{Unb}) \text { and } \mathrm{A}=4 \times\left(7^{2}+5^{2}\right)=296
$$

The nucleus ${ }^{304} \mathrm{Ubn},(\mathrm{Unbilinium})$ was obtained by the reactions:

$$
58 \mathrm{Fe}+249 \mathrm{Bk} \rightarrow 304(123)+3 \mathrm{n} ; \quad 58 \mathrm{Fe}+249 \mathrm{Cf} \rightarrow 304(124)+3 \mathrm{n}
$$

A higher stability of the nucleus with $A=296$ was deducted also by other authors [13], in the form: $\mathrm{Z}=112, \mathrm{~N}=184$, in concordance with recent experiments [14] which gave evidence of the significant increase of the stability of heavy nuclei approaching the magic number $N=184$.

The fact that - at ordinary temperatures, the nuclei with $\mathrm{A}>210$ are generally unstable may indicate either that their real nuclear structure is in accordance with the 'drop' nuclear model, resulted by the rearranging of the nucleons at a specific nuclear temperature $\mathrm{T} \approx 10^{9} \div 10^{11} \mathrm{~K}$ which can 'melt' the initial quasi-crystalline form, or in a vibrational quasi-crystalline state, at a nuclear temperature close to the solid-liquid transition temperature, $\mathrm{T}_{\mathrm{n}}$.

This last possibility is in concordance with the fact that a quasi-crystalline nuclear structure was evidenced by experiments of particle dispersion on heavy cores, (W.Bauer, [15]).

The concordance with the model of alpha-particles cluster type explains also the fact that the decay of the experimentally obtained nuclei, with $\mathrm{Z} \geq 95$ ( $\mathrm{A} \geq 243)$, is generally produced by $\alpha$-particles emission.

Similarly may be formed- according to the model, a nucleus with:

$$
\begin{gathered}
\mathrm{A}=4\left(7^{2}+2 \times 5^{2}\right)=396 ; \quad \mathrm{A}=4 \times\left(2 \times 6^{2}+2 \times 4^{2}\right)=416 ; \quad \mathrm{A}=4 \times\left(2 \times 6^{2}+2 \times 4^{2}+2 \times 2^{2}\right)=448 ; \\
\mathrm{A}=4 \times\left(7^{2}+2 \times 5^{2}+2 \times 3^{2}\right)=468 ; \quad \mathrm{A}=4 \times\left(2 \times 7^{2}+2 \times 5^{2}+2 \times 3^{2}\right)=517 ;
\end{gathered}
$$

Nucleons or alpha-weakly-linked particles formed from valence nucleons may be rotated around the nuclear quasi-crystalline core (Lonnroth, [16]), particularly- as in the extreme- uniparticle model (Schmidt, [17]) by the action of the quantum vortex $\Gamma_{\mu}$ of the nuclear magnetic moment $\mu_{\mathrm{N}}$ (according to the quantum-vortexial nature of the magnetic field, considered in CGT [1-4]) which explains also the nuclear centrifugal potential.

\section{The Explaining of the Nuclear Fission of a Quasi-Crystalline Nucleus by CGT}

The nuclear fission of a quasi-crystalline nucleus may be explained in CGT by a deuteron self-resonance mechanism which generates a decrease in the value of the interaction potential $\mathrm{V}_{\mathrm{n}}$ between nucleons by nucleonic vibrations in portions with incompleteness of the quasi-crystalline nucleonic network or with 
excess nucleons, as consequence of a higher vibration liberty of the nucleons of this nuclear part, according to CGT [1-4].

This mechanism and the energy of the interaction particle (neutron) determine a local change of the quasi-crystalline network into a quasi-liquid phase. This transition is characterized in the nuclear physics by the 'quantality' parameter $\Lambda$ introduced by B. Mottelson [18]:

$$
\Lambda=2\left(\mathrm{~b}_{0} / \mathrm{r}_{0}\right)^{2}=\hbar^{2} / \mathrm{m}_{\mathrm{n}} \mathrm{r}_{0}{ }^{2} \mathrm{~V}_{0}
$$

in which: $\mathrm{r}_{0}$-the inter-particles distance; $\mathrm{m}_{\mathrm{n}}$ - the nucleon mass; $\mathrm{V}_{0}$ - the typical magnitude of the interconstituents interaction.

The parameter $\Lambda$ is defined as the ratio of the zero-point kinetic energy of the confined particle to its potential energy. The liquid phase corresponds to $\Lambda>0.1$, whereas the crystalline solid phase is characterized by values of $\Lambda<0.1$, [19]. In the case of nuclei, using $\mathrm{V}_{0} \approx 100 \mathrm{MeV}$ and $\mathrm{r}_{0} \approx 1 \mathrm{fm}-$ in accordance with the Reid potential, it results $\Lambda \approx 0.5$.

The model also explains the super-asymmetric nuclear fission [20] by the conclusion that the incompleteness of the quasi-crystalline network or a number of excess nucleons leads to a higher 'vibration liberty' $l_{v}$ of these weakly bound nucleons and this vibration decreases the value of the scalar nucleonic potential and determines either the nucleus fission into sub-nuclei with symmetrical quasicrystalline forms (often - "magical" or quasi-stable forms), or gamma vibration spectra, as a result of the self-resonance of less strongly bound nucleons, according to a spin-dependent nuclear potential relation $(\mathrm{CGT},[1-4])$ :

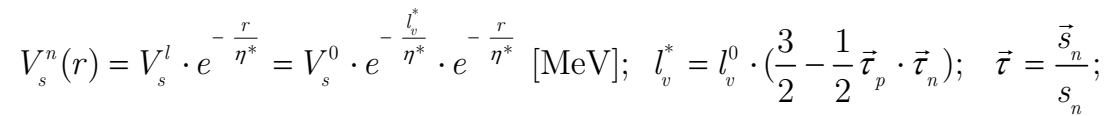

in which: $\mathrm{V}_{\mathrm{s}}{ }^{0}=109.8 \mathrm{MeV} ; \eta^{*}=0.8 \mathrm{fm},[2], l_{v} \cong \mathrm{A}_{\mathrm{v}}$, (the vibration amplitude);

$l_{v}^{0}\left(\mathrm{E}_{\mathrm{v}}\right) \cong 1 \mathrm{fm}$-for deuteron; $\left(l_{v}\left(\mathrm{E}_{\mathrm{v}}=0\right)=0\right) ; l_{v} \sim \mathrm{E}_{\mathrm{v}}=1 / 2 \mathrm{~m}_{\mathrm{n}} \mathrm{V}_{\mathrm{n}}{ }^{2}=1 / 2 \mathrm{k}_{\mathrm{v}} \cdot \mathrm{A}_{\mathrm{v}}{ }^{2}$

The relation (6) corresponds also to the generalized nuclear model by the conclusion that the vibration energy of the nucleons: $\epsilon_{\mathrm{v}} \sim \mathrm{r}^{2}$ decreases the value of the nuclear potential well: $\mathrm{V}_{\mathrm{s}}$.

The degeneration of the nucleonic potential through the nucleon's vibration, according to eqn. (6), may explain also the transformation mechanism of the compound nucleus by interaction with low energy particles up to $2 \mathrm{MeV}$, as in the case of the $\mathrm{Be} 9$ nucleus that can be transformed with a gamma quantum of only $1.78 \mathrm{MeV}$, even if the binding energy given by the sum of its nucleons is $\sim 58 \mathrm{MeV}$. Some reactions with thermal neutrons (of several tens of $\mathrm{eV}$ ) can also be explained as in the case of the reaction of a light $\mathrm{Be} 8$ compound nucleus forming:

a) $\mathrm{Li} 7+\mathrm{H} 1 \rightarrow \mathrm{Be} 8 \rightarrow 2 \mathrm{He} 4+\gamma$ - generated with only $125 \mathrm{eV}$ proton energy, in which Be8 emits an electromagnetic $\gamma$ radiation of $17 \mathrm{MeV}$, or of type $(n ; \alpha)$ like in the reaction:

b) $\mathrm{B} 10+\mathrm{n} \rightarrow \mathrm{Li} 7+\alpha$, generated with thermal neutrons (of few $\mathrm{eV}$ ) even if normally there are necessary neutrons with the energy of $0.5 \ldots 10 \mathrm{MeV}$, [21].

Other exo-energetic nuclear reactions produced at low energies of interaction particles, which sustains the conclusion of CGT regarding the decrease of the scalar potential of the nucleons with relatively small vibration energies, are:

c) ${ }^{2} \mathrm{H}+{ }^{2} \mathrm{H} \rightarrow{ }^{3} \mathrm{He}+\mathrm{n} ; \mathrm{Q}=3.25 \mathrm{MeV}$;

d) ${ }^{2} \mathrm{H}+{ }^{3} \mathrm{H} \rightarrow{ }^{4} \mathrm{He}+\mathrm{n} ; \mathrm{Q}=17.6 \mathrm{MeV}$

in which the resulting neutron energy is 2,5 and $14 \mathrm{MeV}$ respectively and the energy of the incident deuterons may be below $1 \mathrm{MeV}$, for example: $0.1 \mathrm{MeV}$, as in the case d).

According to the model, is explained- by the relation (6) and the self-resonance mechanism of the deuteronic systems, also the emission of a smaller nucleus by a compound nucleus excited with interaction particles of only $1 \div 2 \mathrm{MeV}$ after a time of about $10^{-15} \div 10^{-15}$ seconds, (much longer than the nuclear interaction time: $\sim 10^{-22} \mathrm{~s}$.), in nuclear reactions such as:

$$
{ }^{27} \mathrm{Al}+\mathrm{p} \rightarrow{ }^{24} \mathrm{Mg}+\alpha ; \quad{ }^{4} \mathrm{He}+{ }^{14} \mathrm{~N} \rightarrow{ }^{18} \mathrm{~F}^{*} \rightarrow{ }^{17} \mathrm{~F}+\mathrm{n}
$$

The quasi-crystalline model is in concordance also with the vibrated rigid rotator (of Schmidt type, with the unpaired nucleon generating the spin and the magnetic moment of the nucleus) and with the experiments of alpha-particles scattering on heavy cores, which revealed a nuclear behavior according to a quasi-crystalline nuclear model, (V. Ershov, Alma-Ata [22]).

The correspondence of the nuclear binding energy value experimentally determined with the value resulted by the Weizsäcker's relation [23] which considers a "drop" nuclear model, may be explained by 
the proposed quasi-crystalline model by the conclusion that- at the nuclear temperature $T_{n}$ of solid $\rightarrow$ liquid transition $(\Lambda>0.1)$ induced by the interaction energy with an atomic particle, the nucleons vibrations determines a decreasing of the binding energy according to eqn. (6) and a re-arranging of the nucleons in a more spherically symmetric way, corresponding to a viscous "liquid" phase.

\section{The Possibility of a Cold Genesis of Quasi-Crystalline Nuclei}

A possible explanation of the concordance of the nuclear 'magicity' with the quasi-crystalline nuclear model could be the 'cold' producing of atomic nuclei in the beginning of the Universe' forming, from neutral clusters of four neutrons: $\alpha^{0}=4 n^{0}$, ("tetra-neutron"), the nuclei being formed by beta transformation of at most a half of neutrons into protons during the formation of square forms of neutral $\alpha^{0}$ - clusters and the addition of new $\alpha^{0}$-clusters, this process generating initially small square forms with $2^{2} \alpha^{0}$ particles in which the neutral $\alpha^{0}$ - particles are transformed into double charged $\alpha^{2+}$ particles, attracting thereafter new $\alpha^{0}$-particles, the process being repeated until the forming of double magic nuclei with $\mathrm{Z}=\Sigma\left(2 \mathrm{n}^{2}\right), \mathrm{A}=\Sigma\left(4 \mathrm{n}^{2}\right)$, which may attract excess nucleons or $\alpha^{0}$ - clusters which are partially transformed thereafter into $\alpha^{+}$- clusters.

This cold genesis scenario is, sustainable through a neutron-type "dynamide" model, with partially included negatron in the proton quantum volume (CGT [1-4]), by the fact that -if the neutrons may be formed at $\mathrm{T} \rightarrow 0 \mathrm{~K}$ by a cold gas of protons and electrons and the neutron's stability is increased with the temperature decreasing, the impulse of the formed neutrons may be enough low for be favored the forming of a Bose-Einstein condensate, if the density of neutrons is enough high for the producing of quantum effects, (i.e. - if the thermal de Broglie wavelength: $\lambda_{t}=\hbar / \sqrt{2 m k_{B} T}$ becomes comparable with the average inter-particle spacing).

In 2016, physicists working at the RIKEN nuclear-physics lab in Japan found evidence for the tetraneutron forming in an experiment that involved "firing" neutron-rich helium-8 nuclei at a helium- 4 target [24]. The theoretical calculations indicated a larger energy width than that measured at RIKEN, corresponding to a shorter lifetime [25].

The possibility of neutrons forming instead of hydrogen atoms forming at $\mathrm{T} \rightarrow 0 \mathrm{~K}$ is indirectly sustained also by the experiments of atomic Bose-Einstein condensate collapsing [26] which evidenced a remnant condensate as fraction of the initial condensate and the disappearing of a part of the collapsed BEC. We may suppose logically that the missing part of atoms is explained by the atomic electrons coupling, with the forming of Cooper pairs and their falling to the nucleus, with the transforming of the nuclear protons into neutrons and the releasing of neutral $\alpha^{0}$-clusters.

According to the model, at a temperature close to $0 \mathrm{~K}$, the nucleons can be formed from protons and electrons captured and incorporated into the proton quantum volume, according to the reaction:

$$
{ }^{1} \mathrm{p}_{1}+\mathrm{e}^{-} \rightarrow{ }^{1} \mathrm{n}_{0}+\mathrm{v}_{\mathrm{e}}
$$

with a lifetime higher than that observed at ordinary temperatures (about 15 minutes).

If the neutron's transformation results as in case of the "dynamide" model of CGT, by the neutron's self-resonance given by the oscillation of the neutron negatron' kernel in report with the proton center's position [2-5], we may suppose that- at very low temperature $\mathrm{T} \rightarrow 0 \mathrm{~K}$, the neutron' negatrons $\mathrm{e}^{*}$ remains attached to the proton's volume $\mathrm{p}^{+}$and the $\alpha^{0}$-clusters remain un-transformed until their mutual impact, their coupling resulting as possible by the nuclear force.

In this way they can form quadratic neutral clusters $\alpha^{0}=4 \mathrm{n}^{0}$ by collisions at low energy, the protonic centers of the $\alpha^{0}$ - clusters being linked both by nuclear and magnetic forces, with antiparallel magnetic moments. Also, they are electrically and magnetically coupled with one or two electrons (magnetically coupled negatrons, forming a Cooper pair) positioned in the middle of the square shape $\alpha^{0}=4 n^{0}$, the other two neutronic negatrons being located diametrically opposed to the Cooper pair of negatrons. These marginal electrons can be relatively readily "lost" in the form of beta radiation $\left(\beta^{-}\right)$by electrostatic repulsion between them and similar negatrons of other clusters $\alpha^{0}=4 n^{0}$ with which they come in contact and by vibrations of deuteronic self-resonance. In this way, the clusters $\alpha^{0}$ generating square forms $\mathrm{A}^{0}{ }_{\mathrm{i}}=\mathrm{N}^{2} \alpha^{0}$ are transformed into $\alpha^{+}$- particles, in a time at most equal to the life of the free neutron, ( $\tau \leq \tau_{\mathrm{n}} \approx 15 \mathrm{~min}$.). 
The resulted square forms of $\alpha^{+}$-particles: $\mathrm{A}^{+}=\mathrm{N}^{2} \alpha^{+}$or $\mathrm{A}^{+}=2 \mathrm{~N}^{2} \alpha^{+}$, (doublet), attracts new neutron clusters $\alpha^{0}=4 n^{0}$ which generates two new square forms $A^{0}(n)=n^{2} \alpha^{0},(n \leq N)$, of dimension equal to or lower than the initial one $\left(\mathrm{A}^{0} \mathrm{i}(\mathrm{N})\right)$ and positioned to its square faces.

The process can be repeated with the formation of new square forms $A^{0}\left(n^{\prime}\right),\left(n^{\prime} \leq n\right)$, which then are converted into $\mathrm{A}^{+}(\mathrm{n}$ ') forms.

The ratio N/Z of nuclear stability is given according to the semi-empiric Weizsäcker's relation of the nuclear binding energy [23], in the form:

$$
\mathrm{N} / \mathrm{Z} \approx 1+\left(\mathrm{a}_{\mathrm{c}} / 2 \mathrm{a}_{\mathrm{A}}\right) \cdot \mathrm{A}^{2 / 3}
$$

in which: $\mathrm{a}_{\mathrm{c}} \approx 0.711 ; \mathrm{a}_{\mathrm{A}}=23.7$.

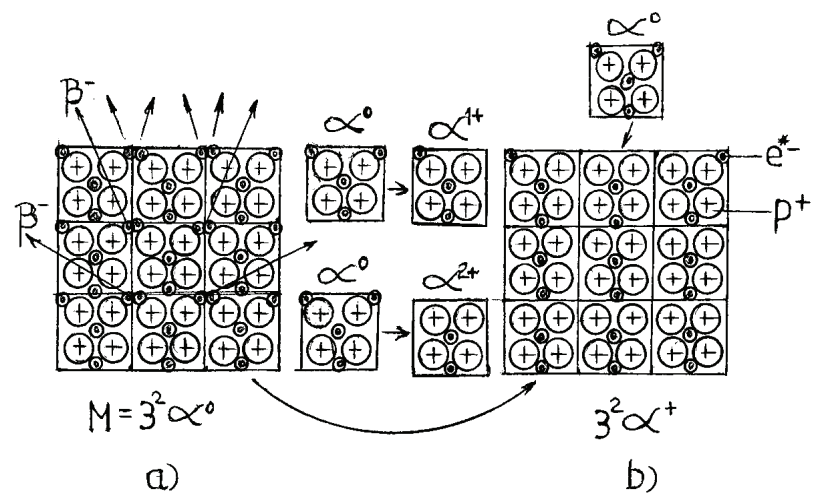

Figure 2. The forming of a nuclear quasi-crystalline form of $\alpha^{0}$-clusters by the forming of a first square form of $\alpha^{0}$ -clusters a) and its transforming into a nuclear square form b)

The dependence of the $\mathrm{a}_{\mathrm{c}}$-coefficient on the Coulomb interaction between protons and on the number $\mathrm{A}^{2 / 3}$ of nucleons positioned in the nuclear surface indicates that the excess of neutrons relative to the number of protons increases with the number of nucleons in the surface of the nucleus.

For the nuclei with N/Z>1 and "magic" A-number, the relation (9) can be explained by the neutron "dynamide' model by the conclusion that a higher positive nuclear charge determines the retaining of peripheral neutronic electrons in the attracted clusters $\alpha^{0}$ with a probability $\mathrm{P} \sim \mathrm{Z}$, these $\alpha^{0}$-clusters being only partially transformed into $\alpha^{2+}$ particles, (partially being transformed into $\alpha^{1+}$-particles), a constant number $\mathrm{C}_{0} \sim\left(\mathrm{a}_{\mathrm{C}} / 2 \mathrm{a}_{\mathrm{A}}\right)$ of $\alpha^{1+}$ - clusters with the neutrons/protons ratio $\mathrm{n} / \mathrm{z}=3 / 1$ being maintained in the nuclear surface unit- phenomenon which generates a mean ratio: $r_{s}=n_{s} / z_{s}>1$. For the nuclei with $\mathrm{N} / \mathrm{Z}>1$, the conclusion of the explicative model is that the excess neutrons are only in the nuclear surface.

For the nuclei with "magic" A- number, if $\mathrm{K}_{1}^{\alpha}$ and $\mathrm{K}_{2}^{\alpha}$ represent the number of nuclear clusters $\alpha^{2+}$ and of $\alpha^{1+}$-clusters in the nuclear surface, $\mathrm{K}_{2}{ }^{\alpha}$ results by a rewritten form of the relation (9):

$$
\begin{gathered}
\mathrm{N} / \mathrm{Z}=\left(2 \mathrm{~K}_{1}{ }^{\alpha}+3 \mathrm{~K}_{2}{ }^{\alpha}\right) /\left(2 \mathrm{~K}_{1}{ }^{\alpha}+\mathrm{K}_{2}{ }^{\alpha}\right) \approx 1+2 \mathrm{~K}_{2}{ }^{\alpha} / \mathrm{Z}=1+\left(\mathrm{a}_{\mathrm{C}} / 2 \mathrm{a}_{\mathrm{A}}\right) \cdot \mathrm{A}^{2 / 3}, \\
\mathrm{~K}_{2}{ }^{2}=\left(\mathrm{a}_{\mathrm{C}} / 4 \mathrm{a}_{\mathrm{A}}\right) \cdot \mathrm{A}^{2 / 3} \cdot \mathrm{Z},
\end{gathered}
$$

in accordance with the explanatory conclusions of the $\mathrm{K}_{2}{ }^{\alpha}$-number's dependence on the nuclear surface and on the nuclear charge $\mathrm{Z}$.

The rotation of the nucleus takes place according to the relation of Aage Bohr:

$$
\mathrm{E}_{\mathrm{rot}}=\left(\mathrm{p}^{2} / 2 \mathrm{~J}\right)=\mathrm{h}^{2} \mathrm{I}(\mathrm{I}+1) / 2 \mathrm{~J}
$$

where $\mathrm{p}$ is the angular momentum, $\mathrm{J}$ is the moment of inertia and $\mathrm{I}$ is the quantum number of the angular momentum, which is an integer for a nucleus with an even number of nucleons and an integer plus a half unit for a nucleus with an odd number of nucleons.

For the nuclei with $\mathrm{N} / \mathrm{Z}>1$ and "magic" Z- number, the excess nucleons are rotated - at least in part, around the double magic kernel with $\mathrm{N}=\mathrm{Z}$, in accordance also with the generalized nuclear model. The property of 'inert kernel' with quasi-null magnetic moment of the quasi-crystal nuclear form is explained by the anti-parallel coupling of the nucleonic magnetic moments in the component $\alpha$-particles. 
The proposed model not exclude the possibility of other quasi-crystalline semi-"magic" nuclear clusters forming, with triangular symmetry, cold formed as neutral clusters of $\mathrm{t}^{0}=3$ neutrons, transformed later into $\mathrm{t}^{+}$clusters (tritium clusters), such as:

$-{ }^{6} \mathrm{Li},(\mathrm{A}=2 \times 3)$ - stable isotope; ${ }^{9} \mathrm{Be},(\mathrm{A}=3 \times 3)$ - the most stable/abundant isotope, or as triangles with 10 nucleons, in the ground state:

${ }^{20} \mathrm{Ne},(\mathrm{A}=2 \times(4+3+2+1))$ - the most stable/ abundant isotope, or with pentagonal symmetry, with $\mathrm{n} \times 5 \times 10$ nucleons, for example:

${ }^{100} \mathrm{Ru}$ (stable/abundant isotope); ${ }^{150} \mathrm{Sm}$-stable/ab. isotope; ${ }^{200} \mathrm{Hg}$ - stable/abundant isotope.

and with hexagonal symmetry, with a stability close to those of the quasi-crystalline forms with tetragonal symmetry and depending also on the nucleus deformability, the initial $\alpha^{0}$-clusters having a rhomboidal form inside a nuclear hexagonal form, in this case (figure 3 ).

The mass number A of a hexagonal quasi-crystalline nuclear form results by the relation:

$$
\mathrm{A}=\Sigma\left(3 \times 4 \times \mathrm{n}^{2}\right) ;(\mathrm{n}=1,2 \ldots 5)
$$

$\mathrm{n}=1 \Rightarrow \mathrm{K}_{\mathrm{a}}=1, \mathrm{~A}=12$, ( ${ }^{12} \mathrm{C}$-the most stable/ab. is. $) ; \mathrm{K}_{\mathrm{a}}=2, \mathrm{~A}=24,\left({ }^{24} \mathrm{Mg}\right.$-the most stable/ab. is. $)$

$\mathrm{n}=2 \Rightarrow \mathrm{A}=48,\left({ }^{48} \mathrm{Ti}\right.$-the most stable isotope); $\mathrm{K}_{\mathrm{a}}=2, \mathrm{~A}=84,\left({ }^{84} \mathrm{Kr}\right.$ - the most stable/ab. is.);

$\mathrm{K}_{\mathrm{a}}=3, \mathrm{~A}=132$, ( ${ }^{132} \mathrm{Xe}-$ the most stable/ab. is.); $\mathrm{K}_{\mathrm{a}}=4, \mathrm{~A}=180,\left({ }^{180} \mathrm{Hf}-\right.$ the most stable/ab. is.);

$\mathrm{n}=3 \Rightarrow \mathrm{A}=108,\left({ }^{108} \mathrm{Pd}\right.$-stable isotope $) ; \mathrm{n}=4 \Rightarrow \mathrm{A}=192,\left({ }^{192} \mathrm{Os}\right.$-stable isotope);

$\mathrm{A}=\left(2 \times 3 \times 4 \times 1^{2}+3 \times 4 \times 2^{2}\right)=72,\left({ }^{72} \mathrm{Ge}-\right.$ the most stable/ab. isotope $)$;

$\mathrm{A}=\left(2 \times 3 \times 4 \times 1^{2}+2 \times 3 \times 4 \times 2^{2}\right)=120,\left({ }^{120} \mathrm{Sn}\right.$ - the most stable/ab. isotope $)$;

$\mathrm{A}=\left(2 \times 3 \times 4 \times 2^{2}+3 \times 4 \times 3^{2}\right)=204,\left({ }^{204} \mathrm{~Pb}\right.$-stable isotope $)$;

$\mathrm{n}=5 \Rightarrow \mathrm{A}=300$ ( ${ }^{300}$ Ununhexium $) ; \mathrm{A}=3 \times 4 \times 4^{2}+2 \times 3 \times 4 \times 3^{2}=408$ (?)

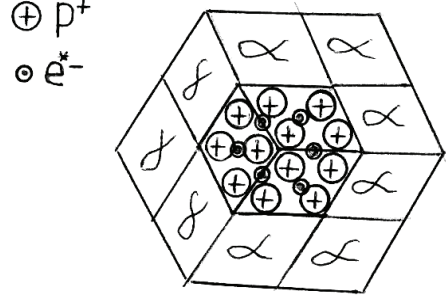

a)

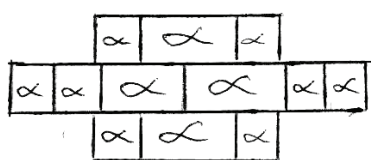

b)

Figure 3. The forming of a quasi-crystalline nucleus from hexagonal forms of $\alpha$-clusters; (72Ge)

The total binding energy of a quasi-crystalline nucleus may be expressed by an equation of BetheWeizsäcker type with the difference that the surface term: $V_{S}=\sigma_{s} \cdot S_{a}=a_{s} A^{2 / 3},\left(a_{s} \approx 17.8 M e V\right)$ is given by the specific quasi-crystalline form of the nucleus, in the form: $V_{s}{ }^{\prime}=\sigma^{\prime} \cdot S_{q}$, the volume term being: $\mathrm{V}_{\mathrm{v}}{ }^{\prime}=\mathrm{a}_{\mathrm{v}}{ }^{\prime} \cdot \mathrm{A}$ with $\mathrm{a}_{\mathrm{v}}{ }^{\prime} \geq \mathrm{a}_{\mathrm{v}} \approx 15.75 \mathrm{MeV}$.

Because $\sigma=(1 / 2) \mathrm{F}_{1} / \mathrm{l}$, (force rectangular of unit lenght), it seems logical to have for $\mathrm{V}_{\mathrm{S}}: \sigma \approx \mathrm{a}_{\mathrm{v}} / 4 \pi \mathrm{r}_{0}^{2}$, $\left(\mathrm{r}_{0} \approx(1.2 \div 1.25) \mathrm{fm}\right.$ - the nucleon's radius resulted from the nuclear radius expression: $\left.\mathrm{R}_{\mathrm{n}} \approx \mathrm{r}_{0} \cdot \mathrm{A}^{1 / 3}\right)$.

The fact that $a_{s}$ is higher than $a_{v}$ in the Weizsäcker's formula indicates either a higher value of $S_{a}$ than those resulted from the 'drop' model or a higher vibration energy of the nucleons in the $S_{a}$-surface than that of the internal nucleons, or both situations.

For a 'magic' or semi-magic quasi-crystalline form, the nuclear mass and the $S_{a}$-surface may be calculated by considering three crystalline levels: a., b. and c. of squared or hexagonal forms of $\alpha$ particles on each upper or lower part of the nucleus (fig. 1), with a number: $\mathrm{n}_{\mathrm{a}}{ }^{2}, \mathrm{n}_{\mathrm{b}}{ }^{2}$ and $\mathrm{n}_{\mathrm{c}}{ }^{2}$ of $\alpha$ particles on each level of squared forms (and $3 \mathrm{n}_{\mathrm{a}}{ }^{2}, 3 \mathrm{n}_{\mathrm{b}}{ }^{2}$ and $3 \mathrm{n}_{\mathrm{c}}{ }^{2}$ for each hexagonal form), $\mathrm{n}_{\mathrm{a}}, \mathrm{n}_{\mathrm{b}}$ and $\mathrm{n}_{\mathrm{c}}$ being the number of $\alpha$-particles on the side length $l_{q}$ of the squared or hexagonal form:

$$
\mathrm{M}_{\mathrm{q}}^{\mathrm{s}}=\mathrm{m}_{\alpha} \cdot\left(\mathrm{K}_{\mathrm{a}} \cdot \mathrm{n}_{\mathrm{a}}{ }^{2}+\mathrm{K}_{\mathrm{b}} \cdot \mathrm{n}_{\mathrm{b}}{ }^{2}+\mathrm{K}_{\mathrm{c}} \cdot \mathrm{n}_{\mathrm{c}}{ }^{2}\right) ; \mathrm{M}_{\mathrm{q}}{ }^{\mathrm{h}}=\mathrm{m}_{\alpha} \cdot 3\left(\mathrm{~K}_{\mathrm{a}} \cdot \mathrm{n}_{\mathrm{a}}{ }^{2}+\mathrm{K}_{\mathrm{b}} \cdot \mathrm{n}_{\mathrm{b}}{ }^{2}+\mathrm{K}_{\mathrm{c}} \cdot \mathrm{n}_{\mathrm{c}}{ }^{2}\right)
$$

with: $\mathrm{K}_{\mathrm{a}}=(1 ; 2 ; 3),\left(\mathrm{K}_{\mathrm{b}}, \mathrm{K}_{\mathrm{c}}\right)=(0 ; 1 ; 2)$ - the total number of squared or hexagonal forms on the levels a., b., c. and:

$$
\mathrm{S}_{\mathrm{a}}=\mathrm{S}_{\mathrm{a}}{ }^{\mathrm{u}}+\mathrm{S}_{\mathrm{a}}{ }^{1}=\mathrm{S}_{\alpha} \cdot 2 \mathrm{n}_{\mathrm{a}}{ }^{2}+1 / 2\left(\mathrm{~s}_{\alpha}\right) \cdot 4\left(\mathrm{~K}_{\mathrm{a}} \cdot \mathrm{n}_{\mathrm{a}}+\mathrm{K}_{\mathrm{b}} \cdot \mathrm{n}_{\mathrm{b}}+\mathrm{K}_{\mathrm{c}} \cdot \mathrm{n}_{\mathrm{c}}\right)=\mathrm{S}_{\alpha} \cdot 2\left(\mathrm{n}_{\mathrm{a}}{ }^{2}+\mathrm{K}_{\mathrm{a}} \cdot \mathrm{n}_{\mathrm{a}}+\mathrm{K}_{\mathrm{b}} \cdot \mathrm{n}_{\mathrm{b}}+\mathrm{K}_{\mathrm{c}} \cdot \mathrm{n}_{\mathrm{c}}\right)
$$

( $\mathrm{s}_{\alpha}$-the surface of the $\alpha$-particle), for a nucleus formed by quasi-crystalline squared forms and: 


$$
\mathrm{S}_{\mathrm{a}}=\mathrm{S}_{\mathrm{a}}{ }^{\mathrm{u}}+\mathrm{S}_{\mathrm{a}}{ }^{1}=\mathrm{s}_{\alpha} \cdot 6 \mathrm{n}_{\mathrm{a}}{ }^{2}+\frac{1}{2}\left(\mathrm{~s}_{\alpha}\right) \cdot 6\left(\mathrm{~K}_{\mathrm{a}} \cdot \mathrm{n}_{\mathrm{a}}+\mathrm{K}_{\mathrm{b}} \cdot \mathrm{n}_{\mathrm{b}}+\mathrm{K}_{\mathrm{c}} \cdot \mathrm{n}_{\mathrm{c}}\right)=\mathrm{s}_{\alpha} \cdot 3\left(2 \mathrm{n}_{\mathrm{a}}{ }^{2}+\mathrm{K}_{\mathrm{a}} \cdot \mathrm{n}_{\mathrm{a}}+\mathrm{K}_{\mathrm{b}} \cdot \mathrm{n}_{\mathrm{b}}+\mathrm{K}_{\mathrm{c}} \cdot \mathrm{n}_{\mathrm{c}}\right)
$$

for a nucleus formed by quasi-crystalline hexagonal forms, with $\mathrm{S}_{\mathrm{a}}{ }^{\mathrm{u}}$-the (upper +lower) surface, $\mathrm{S}_{\mathrm{q}}{ }^{1}$-the lateral surface (geometrically considered, taking of two times the surface of corner nucleons).

The most stable quasi-crystalline forms are those with the high close to the side length value $l_{n}$ and with double symmetry, i.e. -in report with a symmetry axis $\mathrm{x}-\mathrm{x}$, when $\left[\left(\mathrm{n}_{\mathrm{a}}-\mathrm{n}_{\mathrm{b}}\right) ;\left(\mathrm{n}_{\mathrm{b}}-\mathrm{n}_{\mathrm{c}}\right)\right]=2 \mathrm{k},(\mathrm{k}=1,2 \ldots)$ and in report with a symmetry plane $\mathrm{P}$ rectangular to the $\mathrm{x}-\mathrm{x}$ ' axis.

For example, for ${ }^{280} \mathrm{Rg}, \mathrm{A}=4 \times\left(2 \times 5^{2}+2 \times 3^{2}+2 \times 1^{2}\right)=280$ and:

$$
\mathrm{S}_{\mathrm{\alpha}}=\mathrm{s}_{\alpha} \cdot 2\left(5^{2}+2 \times 5+2 \times 3+2 \times 1\right)=\mathrm{s}_{\alpha} \cdot \mathrm{A}_{\mathrm{s}}=\mathrm{s}_{\alpha} \cdot 86,
$$

approximating that: $\mathrm{s}_{\mathrm{r}} \approx 4 \mathrm{~s}_{\mathrm{n}}=4 \pi \mathrm{r}_{0}{ }^{2}$ with $\mathrm{r}_{0} \approx(1.2 \div 1.25) \mathrm{fm}$, with $\sigma_{\mathrm{s}}=\mathrm{a}_{\mathrm{s}} / 4 \pi \mathrm{r}_{0}^{2}=1.03 \mathrm{MeV} / \mathrm{fm}^{2}$ and $\mathrm{R}_{\mathrm{n}}=\mathrm{r}_{0} \mathrm{~A}^{1 / 3}-$ the nucleus' radius, it results that:

$$
\mathrm{V}_{\mathrm{S}}=4 \pi \mathrm{r}_{0}^{2} \mathrm{~A}^{2 / 3} \sigma_{\mathrm{s}}=\mathrm{S}_{\alpha} \cdot \mathrm{A}^{2 / 3} \sigma_{\mathrm{s}}=4 \pi \mathrm{R}_{\mathrm{n}}^{2} \sigma_{\mathrm{s}}=\mathrm{S}_{\mathrm{n}} \cdot \sigma_{\mathrm{s}} ; \mathrm{V}_{\mathrm{s}}{ }^{\prime}=\sigma^{\prime} \cdot \mathrm{S}_{\mathrm{a}}=\mathrm{S}_{\alpha} \cdot \mathrm{A}_{\mathrm{s}} \cdot \sigma^{\prime} ;
$$

For a quasi-crystal state, at the solid $\rightarrow$ liquid transition, characterized by a mean vibration energy $\boldsymbol{\varepsilon}_{\mathrm{v}}{ }^{0}$ of the nucleons, according to the model we may approximate that $\mathrm{a}_{\mathrm{v}}{ }^{\prime}\left(\varepsilon_{\mathrm{v}}{ }^{0}\right) \approx \mathrm{a}_{\mathrm{v}}$ and:

$$
\sigma_{\mathrm{s}}{ }^{\prime}\left(\varepsilon_{\mathrm{v}}{ }^{0}\right)=\mathrm{f}_{\mathrm{v}}{ }^{0} \cdot \sigma_{\mathrm{a}}{ }^{\prime} \approx \mathrm{f}_{\mathrm{v}}{ }^{0} \cdot \mathrm{a}_{\mathrm{v}} / 4 \pi \mathrm{r}_{0}{ }^{2}=\mathrm{f}_{\mathrm{v}}{ }^{0} \cdot \sigma_{\mathrm{a}}=\mathrm{f}_{\mathrm{v}}{ }^{0} \cdot \mathrm{k}_{\sigma} \cdot \sigma_{\mathrm{s}} ;\left(\mathrm{k}_{\sigma}=\mathrm{a}_{\mathrm{v}} / \mathrm{a}_{\mathrm{s}}\right) ; \mathrm{V}_{\mathrm{s}}{ }^{\prime}\left(\varepsilon_{\mathrm{v}}{ }^{0}\right) \rightarrow \mathrm{V}_{\mathrm{S}},
$$

resulting that:

$$
\mathrm{V}_{\mathrm{S}} / \mathrm{V}_{\mathrm{s}}{ }^{\prime} \rightarrow 1 \Rightarrow\left[1 /\left(\mathrm{f}_{\mathrm{v}}{ }^{0} \cdot \mathrm{k}_{\sigma}\right)\right] \cdot\left(\mathrm{A}^{2 / 3} / \mathrm{A}_{\mathrm{s}}\right)=\left[1 /\left(\mathrm{f}_{\mathrm{v}}{ }^{0} \cdot \mathrm{k}_{\sigma}\right)\right] \cdot \mathrm{k}_{\mathrm{A}} \rightarrow 1 ; \mathrm{f}_{\mathrm{v}}{ }^{0}=\mathrm{f}_{\mathrm{v}}\left(\varepsilon_{\mathrm{v}}{ }^{0}\right)
$$

Conform to eqn. (19), an increased ratio $\mathrm{k}_{\mathrm{A}}$ gives an increased binding energy, (corresponding to a lower value of $\mathrm{f}_{\mathrm{v}}{ }^{(}\left(\varepsilon_{\mathrm{v}}{ }^{0}\right)$ ) so a nucleus with a lower value of $\mathrm{k}_{\mathrm{A}}$ may be 'melted' with a lower vibration energy.

For the previous case, $(A=280)$ it results that: $A^{2 / 3}=42.8 ; A_{s}=86$, and $k_{A}=\left(A^{2 / 3} / A_{s}\right) \approx 0.49$.

For $\mathrm{A}=4 \times\left(4 \times 3^{2}\right)=144,\left({ }^{144} \mathrm{Ce}\right.$ - stable isotope $)$, the quasi-crystalline nucleus has an almost cubic form, which may become spherical for $\Lambda>0.1$, (at more intense vibrations of the nucleons), and we have:

$$
\mathrm{A}^{2 / 3}=27.47 ; \mathrm{S}_{\mathrm{a}}=\mathrm{S}_{\alpha} \cdot \mathrm{A}_{\mathrm{s}}=\mathrm{S}_{\alpha} \cdot\left(2 \times 3^{2}+2 \times 4 \times 3\right)=\mathrm{S}_{\alpha} \cdot 42 ;\left(\mathrm{A}^{2 / 3} / \mathrm{A}_{\mathrm{s}}\right) \approx 0.654 .
$$

The ratio $k_{A}=\left(A^{2 / 3} / A_{s}\right)$ may be considered a quasi-crystalline stability parameter.

For example, if will be extracted an $\alpha$-particle from the corner of a 'magic' quasi-crystalline form:

$$
4 \times\left(4 \times 3^{2}\right)=144\left({ }^{144} \mathrm{Ce}\right) \text {, }
$$

we will have: $\mathrm{A}=4 \times\left(4 \times 3^{2}\right)=144-\alpha=140\left({ }^{140} \mathrm{Ce}-\right.$ stable isotope $)$, and:

$$
\mathrm{A}^{2 / 3}=26.96 ; \mathrm{S}_{\mathrm{a}}=\mathrm{S}_{\alpha} \cdot \mathrm{A}_{\mathrm{s}}=\mathrm{S}_{\alpha} \cdot\left(2 \times 3^{2}+2 \times 4 \times 3\right)=\mathrm{S}_{\alpha} \cdot 42 ;\left(\mathrm{A}^{2 / 3} / \mathrm{A}_{\mathrm{s}}\right) \approx 0.642<0.654
$$

and if the $\alpha$-particle is extracted from the edge, we will have:

$$
\mathrm{S}_{\mathrm{a}}=\mathrm{S}_{\alpha} \cdot \mathrm{A}_{\mathrm{s}}=\mathrm{S}_{\alpha} \cdot\left(2 \times 3^{2}+2 \times 4 \times 3+1\right)=\mathrm{S}_{\alpha} \cdot 43 ;\left(\mathrm{A}^{2 / 3} / \mathrm{A}_{\mathrm{s}}\right) \approx 0.627<0.642,
$$

the physical explanation being the fact that the generated hole increases the vibration liberty $l_{v}$ of the adiacent remained nucleons, decreasing their binding energy, according to the relation (6).

So, by the parameter $\mathrm{k}_{\mathrm{A}}$ it may be verified that the adding of $\alpha$-particles to a 'magic' quasi-crystalline or extracting $\alpha$-particles form it gives a nucleus less stable than the initial nucleus, explaining the fact that the isotopes corresponding to a complete quasi-crystalline nuclear form are generally more stable than the adjacent isotopes or at least stable isotopes.

The maintaining of the quasi-crystalline form during the nucleus forming may be explained by the conclusion that a lower vibration energy of the nucleons: $\varepsilon_{\mathrm{v}}<\varepsilon_{\mathrm{v}}{ }^{0}$ permitted a higher mean value of the binding energy per nucleon, generated by a lower inter-distance between nucleons closer to the maximal value of the interaction potential $\left(d_{i} \rightarrow 0.9 \mathrm{fm}\right)$, in accordance with the eqn. (6) and with the criterion: $\Lambda<0.1$ resulted by eqn. (5)).

According to eqn. (6), because the nucleons of the quasi-crystal nucleus edge have a higher vibration liberty $l_{v}$, they decrease the nucleus binding energy more than the nucleons of the upper/lower surface, in a proportion: $\mathrm{f}_{\mathrm{v}}{ }^{\prime}\left(\varepsilon_{\mathrm{v}}\right) \approx \mathrm{k}_{0} \cdot\left(\mathrm{S}_{\mathrm{q}}{ }^{1} / \mathrm{S}_{\mathrm{q}}\right)=\mathrm{k}_{0}\left(\mathrm{~A}_{\mathrm{l}} / \mathrm{A}_{\mathrm{s}}\right) ;\left(\mathrm{A}_{\mathrm{l}}=\mathrm{S}_{\mathrm{q}}{ }^{1} / \mathrm{s}_{\alpha} ; \mathrm{k}_{0}\left(\varepsilon_{\mathrm{v}}\right)>1\right)$.

This assumption, resulted by the expression (6) of the nuclear potential, is in concordance with the existence of exotic nuclear reactions such as: $\mathrm{B} 10+\mathrm{n} \rightarrow \mathrm{Li} 7+\alpha$ generated with thermal neutrons instead of neutrons with $0.5 \ldots 10 \mathrm{MeV},\lceil 21\rceil$ and with the fact that the vibration energy formula for nuclei with excess or lack of nucleons in report with the central completed part: $\mathrm{E}=\mathrm{n} \cdot \mathrm{h} \omega, \quad(\mathrm{n}=1,2 \ldots)$ is correspondent to the energy formula of a quantum harmonic oscillator with the basic level: $\mathrm{E}^{0}=\hbar \omega \approx 0.3 \mathrm{MeV}$.

Also, we must consider a dependence of $\mathrm{f}_{\mathrm{v}}\left(\boldsymbol{\varepsilon}_{\mathrm{v}}\right)=\mathrm{f}_{\mathrm{v}} \cdot \Delta \mathrm{f}_{\mathrm{v}}$ on the nucleus deformation:

in the semi-empiric form:

$$
\delta_{\mathrm{n}}=\left(\mathrm{l}_{\mathrm{a}}-\mathrm{h}\right) / \mathrm{l}_{\mathrm{a}}=\left(2 \mathrm{n}_{\mathrm{a}}-\left(\mathrm{K}_{\mathrm{a}}+\mathrm{K}_{\mathrm{b}}+\mathrm{K}_{\mathrm{c}}\right)\right) / 2 \mathrm{n}_{\mathrm{a}}
$$


$\mathrm{f}_{\mathrm{v}}\left(\varepsilon_{\mathrm{v}}\right)=\mathrm{f}_{\mathrm{v}}{ }^{\prime} \cdot \Delta \mathrm{f}_{\mathrm{v}}=\mathrm{K}_{\mathrm{v}} \cdot\left(\mathrm{A}_{\mathrm{l}} / \mathrm{A}_{\mathrm{s}}\right) ; \quad \mathrm{K}_{\mathrm{v}}=\mathrm{k}_{0} \cdot\left(1+\mathrm{k}_{\mathrm{d}} \cdot \delta_{\mathrm{n}}{ }^{2}\right)$

because a higher deformation $\delta_{\mathrm{n}}$ generates- by the nucleus' ground state vibrations (oscillations in report with the initial position, perpendicular on the $\mathrm{x}-\mathrm{x}^{\prime}$ axis), a weakening of the $\mathrm{F}_{1}$-binding force between adjacent nucleons. For ${ }^{144}$ Ce it results that: $\delta_{n}=(2 \cdot 3-4) / 6=1 / 3$.

This assumption is in concordance with the fact that high-mass nuclei have low-lying excited states that are described as vibrations or rotations of non-spherical nuclei.

The expression of the difference $\Delta \mathrm{V}_{\mathrm{q}}=\left(\mathrm{V}_{\mathrm{v}}{ }^{\prime}-\mathrm{V}_{\mathrm{s}}{ }^{\prime}\right) \geq \Delta \mathrm{V}_{\mathrm{n}}=\left(\mathrm{a}_{\mathrm{v}} \cdot \mathrm{A}-\mathrm{a}_{\mathrm{s}} \cdot \mathrm{A}^{2 / 3}\right)$ results in the approximate form:

$$
\Delta \mathrm{V}_{\mathrm{a}}=\left(\mathrm{V}_{\mathrm{v}}{ }^{\prime}-\mathrm{V}_{\mathrm{s}}{ }^{\prime}\right)=\left(\mathrm{a}_{\mathrm{v}}{ }^{\prime} \cdot \mathrm{A}-\sigma_{\mathrm{s}}{ }^{\prime} \cdot \mathrm{S}_{\mathrm{q}}\right)=\left(\mathrm{a}_{\mathrm{v}}{ }^{\prime} \cdot \mathrm{A}-\mathrm{a}_{\mathrm{v}}{ }^{\prime} \cdot \mathrm{K}_{\mathrm{v}} \cdot \mathrm{A}_{\mathrm{l}}\right)
$$

Admitting that at the solid $\rightarrow$ liquid transition we have $\mathrm{a}_{\mathrm{v}}{ }^{\prime} \approx \mathrm{a}_{\mathrm{v}},\left(\sigma_{\mathrm{s}}{ }^{\prime} \approx \mathrm{f}_{\mathrm{v}} \cdot \sigma_{\mathrm{a}}\right)$, we may verify if the relation (21) is plausible by calculating the value of $\mathrm{K}_{\mathrm{v}}$ in the hypothesis: $\Delta \mathrm{V}_{\mathrm{q}}\left(\left(\varepsilon_{\mathrm{v}}{ }^{0}\right) \approx \Delta \mathrm{V}_{\mathrm{n}}\left(\varepsilon_{\mathrm{v}}{ }^{0}\right)\right.$.

For heavier nuclei with quasi-crystalline form closer to a spherical form, such as ${ }^{144} \mathrm{Ce},\left(\mathrm{A}=4 \times\left(4 \times 3^{2}\right)\right.$; $\mathrm{A}_{\mathrm{s}}=42 ; \mathrm{A}_{\mathrm{l}}=24$ ), we have:

$$
\left.\Delta \mathrm{V}_{\mathrm{a}}\left({ }^{144} \mathrm{Ce}\right) \approx \Delta \mathrm{V}_{\mathrm{n}}{ }^{144} \mathrm{Ce}\right)=1779 \mathrm{MeV} ; \Rightarrow \mathrm{a}_{\mathrm{v}} \cdot \mathrm{K}_{\mathrm{v}} \cdot \mathrm{A}_{\mathrm{l}} \approx \mathrm{a}_{\mathrm{s}} \cdot \mathrm{A}^{2 / 3}=489 \mathrm{MeV},\left(\mathrm{A}_{\mathrm{l}}\left({ }^{144} \mathrm{Ce}\right)=24\right) ;
$$

It results the value: $\mathrm{K}_{\mathrm{v}} \approx 1.29$.

We may use the hypothesis: $\mathrm{a}_{\mathrm{v}}{ }^{2} \approx \mathrm{a}_{\mathrm{v}}=15.75, \Delta \mathrm{V}_{\mathrm{q}}\left(\varepsilon_{\mathrm{v}}{ }^{0}\right) \approx \Delta \mathrm{V}_{\mathrm{n}}\left(\varepsilon_{\mathrm{v}}{ }^{0}\right)$ also for a lighter quasi-crystal nucleus: ${ }^{72} \mathrm{Ge}\left(\mathrm{A}=4 \times 2 \times 3^{2}\right)$, for which we have:

$$
\mathrm{V}_{\mathrm{S}}=\mathrm{a}_{\mathrm{S}} \cdot \mathrm{A}^{2 / 3}=308 \mathrm{MeV} ; \Delta \mathrm{V}_{\mathrm{n}}=826 \mathrm{MeV} ; \mathrm{A}_{\mathrm{l}}=12 ; \delta_{\mathrm{n}}=(2 \cdot 3-2) / 6=2 / 3 ; \mathrm{k}_{\mathrm{A}}=0.57 \text {, }
$$

resulting the equations:

$$
\mathrm{K}_{\mathrm{v}}(\mathrm{Ce})=\mathrm{k}_{0} \cdot\left(1+\mathrm{k}_{\mathrm{d}} / 3^{2}\right) \approx 1.29 ; \mathrm{K}_{\mathrm{v}}(\mathrm{Ge})=\mathrm{k}_{0} \cdot\left(1+0 .(6)^{2} \cdot \mathrm{k}_{\mathrm{d}}\right)=308 /(15.75 \times 12) \approx 1.629 .
$$

which gives: $\mathrm{k}_{0}=1.177 ; \mathrm{k}_{\mathrm{d}}=0.865$.

For the heavier nucleus ${ }^{280} \mathrm{Rg}\left(\mathrm{A}=280 ; \mathrm{A}_{\mathrm{s}}=86 ; \mathrm{A}_{\mathrm{l}}=36\right)$, for which we have: $\mathrm{V}_{\mathrm{S}}=17.8 \times 42.8=761.8 \mathrm{MeV}$; $\Delta \mathrm{V}_{\mathrm{n}}=3648 \mathrm{MeV} ; \delta_{\mathrm{n}}=(2 \cdot 5-2 \cdot 3) / 10=0.4$, with the resulted values we obtain:

$\mathrm{V}_{\mathrm{s}}{ }^{\prime}(\mathrm{Rg}) \approx \mathrm{a}_{\mathrm{v}} \cdot \mathrm{k}_{0}\left(1+\mathrm{k}_{\mathrm{d}} \cdot \delta_{\mathrm{n}}{ }^{2}\right) \cdot \mathrm{A}_{\mathrm{l}} \approx 759.7 \mathrm{MeV} ; \Delta \mathrm{V}_{\mathrm{q}} \approx 3650 \mathrm{MeV}, \Delta \mathrm{V}_{\mathrm{q}}$ resulting almost equal with $\Delta \mathrm{V}_{\mathrm{n}}$. So the form (21) of $\mathrm{K}_{\mathrm{v}}$ results as plausible.

If we take into account also the nuclear density lowering with the mass number $\mathrm{A}$, in the form proposed by Myers and Swiatecki [27]:

$$
\begin{gathered}
\Delta \mathrm{V}_{\mathrm{n}}^{\mathrm{s}}=\Delta \mathrm{V}_{\mathrm{n}}{ }^{0} \cdot \mathrm{f}_{\mathrm{I}}=\left(\mathrm{V}_{\mathrm{v}}^{0}-\mathrm{V}_{\mathrm{s}}^{0}\right) \cdot \mathrm{f}_{\mathrm{I}}=\left(\mathrm{c}_{\mathrm{v}} \cdot \mathrm{A}-\mathrm{c}_{\mathrm{s}} \cdot \mathrm{A}^{2 / 3}\right) \cdot\left(1-\mathrm{k}_{\mathrm{v}} \mathrm{I}^{2}\right) ; \\
\left(\mathrm{f}_{\mathrm{I}}=\left(1-\mathrm{k}_{\mathrm{v}} \mathrm{I}^{2}\right) ; \mathrm{I}=(\mathrm{N}-\mathrm{Z}) / \mathrm{A} ; \mathrm{c}_{\mathrm{v}}=15.677 ; \mathrm{c}_{\mathrm{s}}=18.56 ; \mathrm{k}_{\mathrm{v}} \approx 1.79\right),
\end{gathered}
$$

we have: $\mathrm{V}_{\mathrm{s}}^{0}(\mathrm{Ge})=321.2 \mathrm{MeV} ; \mathrm{V}_{\mathrm{s}}^{0}(\mathrm{Ce})=509.8 \mathrm{MeV} ; \mathrm{V}_{\mathrm{s}}^{0}(\mathrm{Rg})=794.3 \mathrm{MeV}$;

$$
\begin{gathered}
\Delta \mathrm{V}_{\mathrm{n}}{ }^{0}(\mathrm{Ge})=807.5 \mathrm{MeV} ; \Delta \mathrm{V}_{\mathrm{n}}{ }^{0}(\mathrm{Ce})=1747.6 \mathrm{MeV} ; \Delta \mathrm{V}_{\mathrm{n}}{ }^{0}(\mathrm{Rg})=3595.2 \mathrm{MeV} \text {, and: } \\
\mathrm{K}_{\mathrm{v}}=\mathrm{k}_{0} \cdot\left(1+\mathrm{k}_{\mathrm{d}} \cdot \delta_{\mathrm{n}}{ }^{2}\right) \leq \mathrm{V}_{\mathrm{s}}{ }^{0} /\left(\mathrm{c}_{\mathrm{v}} \cdot \mathrm{A}_{\mathrm{l}}\right)
\end{gathered}
$$

We may calculate the value $\mathrm{k}_{0}\left({\varepsilon_{\mathrm{v}}}^{0}\right)$ given by a vibration energy ${\varepsilon_{\mathrm{v}}}^{0}$ of nucleons characteristic to the solid $\rightarrow$ liquid transition, using a quasi-crystal nucleus with $\delta_{\mathrm{n}} \approx 0$, (close to the spherical form), such as ${ }^{64} \mathrm{Zn}$, which has: $\mathrm{A}=4 \times 4 \times 2^{2} ; \mathrm{A}_{\mathrm{l}}=2 \times(2 \times 4)=16=\mathrm{A}^{2 / 3} ; \mathrm{V}_{\mathrm{s}}{ }^{0}(\mathrm{Zn})=\mathrm{c}_{\mathrm{s}} \cdot \mathrm{A}^{2 / 3}=296.9 \mathrm{MeV} ; \Delta \mathrm{V}_{\mathrm{n}}{ }^{0}(\mathrm{Zn})=706.4 \mathrm{MeV}$, and for which we reconsider the approximation: $\Delta \mathrm{V}_{\mathrm{q}}\left(\varepsilon_{\mathrm{v}}{ }^{0}\right) \approx \Delta \mathrm{V}_{\mathrm{n}}\left(\varepsilon_{\mathrm{v}}{ }^{0}\right)$, which gives:

$$
\mathrm{k}_{0}\left(\varepsilon_{\mathrm{v}}{ }^{0}\right)=\mathrm{k}_{0}{ }^{\prime} \approx \mathrm{V}_{\mathrm{s}}{ }^{0} /\left(\mathrm{c}_{\mathrm{v}} \cdot \mathrm{A}_{\mathrm{l}}\right)=\mathrm{c}_{\mathrm{s}} / \mathrm{c}_{\mathrm{v}} \approx 1.1839
$$

which is a plausible value because for a quasi-crystal model, we must have: $\mathrm{k}_{0}\left(\varepsilon_{\mathrm{v}}{ }^{0}\right) \leq \mathrm{c}_{\mathrm{s}} / \mathrm{c}_{\mathrm{v}}$.

We must take into account also different values for the constant $k_{v}$ of eqn. (24) when the factor $f_{I}$ decreases the value of $\mathrm{V}_{\mathrm{v}}{ }^{0}$ or of $\mathrm{V}_{\mathrm{s}}{ }^{0}$ [28]. We may use in this case, for $\Delta \mathrm{V}_{\mathrm{q}}$, the semi-empiric form:

$$
\begin{aligned}
\Delta V_{q} & =\Delta V_{q}^{0} \cdot f_{I}=\left(V_{q v}^{0}-V_{q s}^{0}\right) \cdot f_{I}=\left[c_{v}^{\prime} A \cdot\left(1-k_{a} I^{2}\right)-K_{v} \cdot c_{v}^{\prime} \cdot A_{l}\right] \cdot f_{I} \\
& \approx c^{\prime}{ }_{v} A \cdot\left[1-\left(k_{a}+k_{v}\right) \cdot I^{2}\right]-V_{q s}^{0} \cdot\left(1-k_{v} I^{2}\right)[M e V] ; \\
f_{I} & =\left(1-k_{v} I^{2}\right) ; I=(N-Z) / A ; K_{v}=k_{0} \cdot\left(1+k_{d} \cdot \delta_{n}^{2}\right) ; \delta=\left(l_{a}-h\right) / l_{a} ; c_{v}^{\prime} \geq c_{v} ; k_{0}\left(\varepsilon_{v}^{0}\right)=c_{s} / c_{v}
\end{aligned}
$$

Admitting that at the solid $\rightarrow$ liquid transition we have $\mathrm{c}_{\mathrm{v}}{ }^{\prime} \rightarrow \mathrm{c}_{\mathrm{v}}$ and $\Delta \mathrm{V}_{\mathrm{a}} \rightarrow \Delta \mathrm{V}_{\mathrm{n}}$, we may approximate the values of the constants $\mathrm{k}_{\mathrm{d}}\left(\varepsilon_{0}\right)$ and $\mathrm{k}_{\mathrm{a}}\left(\varepsilon_{0}\right)$ by the hypothesis: $\mathrm{c}_{\mathrm{v}}{ }^{2}\left(\varepsilon_{\mathrm{v}}{ }^{0}\right) \approx \mathrm{c}_{\mathrm{v}} ; \Delta \mathrm{V}_{\mathrm{q}}{ }^{0} \approx \Delta \mathrm{V}_{\mathrm{n}}{ }^{0}$ used for ${ }^{72} \mathrm{Ge}$ and ${ }^{280} \mathrm{Rg}$.

With $\mathrm{k}_{0}=\mathrm{c}_{\mathrm{s}} / \mathrm{c}_{\mathrm{v}}$ we obtain the values: $\mathrm{k}_{\mathrm{d}}\left(\varepsilon_{0}\right)=0.9665 ; \mathrm{k}_{\mathrm{a}}\left(\varepsilon_{0}\right)=0.1$. With: $\mathrm{I}^{2}(\mathrm{Zn})=0.0039 ; \mathrm{I}^{2}(\mathrm{Ge})=0.01234$; $\mathrm{I}^{2}(\mathrm{Ce})=0.0378 ; \mathrm{I}^{2}(\mathrm{Rg})=0.0429$, it results:

$$
\Delta \mathrm{V}_{\mathrm{q}}{ }^{0}(\mathrm{Zn})=706 \mathrm{MeV} ; \quad \Delta \mathrm{V}_{\mathrm{q}}{ }^{0}(\mathrm{Ge})=809 \mathrm{MeV} ; \quad \Delta \mathrm{V}_{\mathrm{q}}{ }^{0}(\mathrm{Rg})=3596.5 \mathrm{MeV} ;
$$

- For ${ }^{144} \mathrm{Ce},\left(\delta_{\mathrm{n}}=1 / 3\right)$, with eqn. $(26)$ we obtain: $\mathrm{K}_{\mathrm{v}}(\mathrm{Ce})=\mathrm{k}_{0}\left(1+0.9665 \cdot\left(0 .(3)^{2}\right)=1.311\right.$;

$$
\mathrm{V}_{\mathrm{qv}}{ }^{0}(\mathrm{Ce})=2248.9 \mathrm{MeV} ; \quad \mathrm{V}_{\mathrm{qs}}{ }^{0}(\mathrm{Ce})=\mathrm{K}_{\mathrm{v}} \cdot \mathrm{c}_{\mathrm{v}} \cdot \mathrm{A}_{\mathrm{l}}=493.3 \mathrm{MeV} ; \quad \Delta \mathrm{V}_{\mathrm{q}}{ }^{0}(\mathrm{Ce})=1755.6 \mathrm{MeV} \text {, }
$$


i.e.- with $\sim 0.45 \%$ higher than $\Delta \mathrm{V}_{\mathrm{n}}{ }^{0}(\mathrm{Ce})=1747.6 \mathrm{MeV}$, (obtained with eqn. (24)), as consequence of its "magic" form, given as quasi-crystal of squared forms with $\alpha^{2+}$ and $\alpha^{+}$particles with low deformation $\delta_{n}$. - For ${ }^{160} \mathrm{Dy}$, (stable isotope: $\mathrm{Z}=66 ; \mathrm{A}=4 \times\left(2 \times 4^{2}+2 \times 2^{2}\right)$ ), we have: $\mathrm{A}_{1}=4 \times(4+2)=24 ; \mathrm{I}=0.175$;

$$
\begin{gathered}
\mathrm{V}_{\mathrm{v}}{ }^{0}(\mathrm{Dy})=2508.3 \mathrm{MeV} ; \mathrm{V}_{\mathrm{s}}^{0}(\mathrm{Dy})=547 \mathrm{MeV} ; \Delta \mathrm{V}_{\mathrm{n}}{ }^{0}=1961.3 \mathrm{MeV} ; \delta_{\mathrm{n}}=(2 \cdot 4-4) / 8=1 / 2 ; \\
\mathrm{K}_{\mathrm{v}}=1.47 ; \mathrm{V}_{\mathrm{av}}{ }^{0}(\mathrm{Dy})=2500.6 \mathrm{MeV} ; \mathrm{V}_{\mathrm{as}}{ }^{0}(\mathrm{Dy})=553 \mathrm{MeV} ; \Delta \mathrm{V}_{\mathrm{q}}{ }^{0}(\mathrm{Dy})=1947.6 \mathrm{MeV},
\end{gathered}
$$

the value $\Delta \mathrm{V}_{\mathrm{q}}{ }^{0}$ (Dy) resulting with $0.7 \%$ lower than $\Delta \mathrm{V}_{\mathrm{n}}{ }^{0}$ given by eqn. (24), as consequence of a higher deformation $\delta_{\mathrm{n}}$ of the quasi-crystal nucleus, which generates a higher ground state vibration of the whole nucleus.

It results that the semi-empiric expression (26) for the binding energy of the quasi-crystal nucleus gives plausible indications about the causes of the nucleus' 'melting'. In accordance also with the expression (5) of the 'quantality' parameter, it results that the nucleus can be in a quasi-crystalline state only at low vibration energies, whereas during an interaction with an atomic particle the nucleus becomes at least partially (at least in the impact zone) a quantum liquid state, by nucleons' rearrangement, the mean value: $\sim 8 \mathrm{MeV}$ of the binding energy per nucleon -characteristic to the "drop" model, resulting by ground state vibrations of the nucleons which are higher than those of the quasicrystal nucleus ground state, in accordance with the eqn. (26) and corresponding by eqn. (6) to a vibration liberty' of $\sim 0.8 \mathrm{fm}$ in report with an initial position corresponding to an inter-distance: $\mathrm{r}_{0} \approx 1.25$ fm.

According to the model, this value of the binding energy between two adjacent nucleons may be characteristic to the nucleons of the quasi-crystal nucleus at the solid $\rightarrow$ liquid transition temperature, when the vibration energy of the surface nucleons (mainly-those of the lateral surface, weaker bind) is transmitted to the internal nucleons.

According to the model, the quasi-crystal nucleus 'melting' and its transition to a 'drop' form is realized by an intermediary super-viscous state, in which $\delta_{\mathrm{n}} \rightarrow 0$ and $\mathrm{k}_{0} \approx \mathrm{k}_{0}{ }^{\prime}=\mathrm{c}_{\mathrm{s}} / \mathrm{c}_{\mathrm{v}}$, by the absorption of a specific energy: $\Delta \mathrm{E}_{\mathrm{T}}=\mathrm{E}_{\mathrm{q}}-\mathrm{E}_{\mathrm{n}},\left(\mathrm{E}_{\mathrm{q}}, \mathrm{E}_{\mathrm{n}}\right.$-the binding energy in the solid /liquid state) and by the nucleus' dilation.

Also, it results by the model that the known vibrations spectrum of the stable nuclei with $\mathrm{A}<150$ resulted by surface nucleons vibrations may be specific also to a quasi-crystalline nuclear state, if the energy given by atomic vibrations at ordinary temperature is close to but lower than this 'melting' energy, $\Delta \mathrm{E}_{\mathrm{T}}$.

\section{Conclusions}

It was shown in the paper that the stable nuclei, with "magic" or semi-"magic" number of protons or and neutrons: $2 ; 8 ; 20 ; 28 ;(32,36,40) ; 50 ; 82 ; 126$, are retrieved by a quasi-crystalline nuclear model, of ground state $\mathrm{T} \rightarrow 0 \mathrm{~K}$, as symmetrical quasi-crystalline forms resulted from overlapping of integer number of alpha particles with $2 \mathrm{n}^{2}$ protons and $4 \mathrm{n}^{2}$ nucleons $\left(\mathrm{Z}=\Sigma\left(2 \mathrm{n}^{2}\right) ; \mathrm{n}=1,2, \ldots 7\right)$ with a minimum deformability for the double 'magic' nuclei, which suggests a possible cold genesis of the nuclear matter.

The possibility of nuclear matter cold genesis may be sustained by a dynamide model of neutron resulted from a cold genesis theory, by the hypothesis of $\alpha^{0}$ - neutron clusters cold forming, (at $\left.\mathrm{T} \rightarrow 0 \mathrm{~K}\right)$, by the nuclear forces, and the generating of small square forms with $n^{2} \alpha^{0}$ particles in which the neutral $\alpha^{0}$-particles are transformed into charged $\alpha^{+}$and $\alpha^{2+}$ particles, attracting new $\alpha^{0}$-particles, the process being repeated until the forming of double magic nuclei with $\mathrm{Z}=\Sigma\left(2 \mathrm{n}^{2}\right)$ which may attract excess nucleons or $\alpha^{0}$-clusters (transformed thereafter into $\alpha^{+}$clusters) or of nuclei with "magic" $A=\mathrm{Z}+\mathrm{N}$ number, as in the case of the nucleus ${ }^{208} \mathrm{~Pb}_{82}$ which corresponds to the initial form: ${ }^{208} \mathrm{~N}_{104}\left(\mathrm{Z}=2\left(4^{2}+6^{2}\right)\right)$ in which 22 attracted $\alpha^{0}$-clusters were transformed into $\alpha^{+}$clusters, by $\beta^{-}$radiation emission.

An argument in the favor of the model is the fact that the nuclei resulted as complete quasicrystalline forms with $\mathrm{A}=\Sigma\left(4 \mathrm{n}^{2}\right)$ nucleons are at least stable isotopes, the ratio: $\mathrm{k}_{\mathrm{A}}=\left(\mathrm{A}^{2 / 3} / \mathrm{A}_{\mathrm{s}}\right)$ (with $\mathrm{A}_{\mathrm{s}}=\mathrm{S}_{\mathrm{q}} / \mathrm{s}_{\alpha}$ ) being higher for these nuclei compared with their neighboring isotopes.

The proposed model predicts that the nucleus with $\mathrm{A}=4\left(5^{2}+7^{2}\right)=296$ nucleons and $\mathrm{Z}=114 \div 120$ is more stable than the form: 114/184, $(\mathrm{A}=298)$ predicted with the "nuclear shells" model.

Also, it results as possible cold semi-"magic" nuclei with hexagonal symmetry, with $\mathrm{A}=\Sigma\left(3 \times 4 \times \mathrm{n}^{2}\right)$, $(\mathrm{n}=1 \ldots 5)$. 
The nuclear binding energy of the quasi-crystal nucleus at the solid $\rightarrow$ liquid transition results close to the value given by the Myers and Swiatecki equation by considering its decreasing proportional not only with the nucleus' surface, but also with the ratio: $\left(\mathrm{S}_{\mathrm{r}}{ }^{1} / \mathrm{S}_{\mathrm{n}}\right)$ between the lateral and the total surface of the nucleus- as consequence of a higher vibration energy of the nucleons contained by the lateral surface and with the nucleus deformation: $\delta_{n}=\left(l_{a}-h\right) / l_{a}$ - as consequence of the fact that a higher deformation $\delta_{n}$ generates a weakening of the $\mathrm{F}_{1}$-binding force between adjacent nucleons by the nucleus' ground state vibrations.

It results -according to the model, that the lowering of the nucleons vibration liberty and of the nucleus' oscillation liberty increases the nuclear binding energy, this explaining also the possibility of the quasi-crystal state maintaining at very low temperatures $\mathrm{T} \rightarrow 0 \mathrm{~K}$ and the possibility of a neutron star transforming into a 'black hole' type star, at a critical mass value.

It is known also that the nuclear matter may be compressed and heated also by nuclear shock waves, mechanism which may generate the forming of multi-hyper-nuclei of $\Lambda, \Sigma, \Xi, \Omega$ - particles, according to some studies [29]. Extrapolating the resulted conclusions looking a possible cold genesis of the nuclear matter and the 'dynamide' model of neutron resulted in CGT, it results as possible also the cold forming of hyper-nuclei from positive charged and neutral hyperons $\left(\Lambda^{0}, \Lambda^{+}, \Sigma^{0}, \Sigma^{+}, \Xi^{0}\right)$, with increased lifetime compared with the usual lifetime of the hyperon $\left(\sim 10^{-10} \mathrm{~s}\right)$, as consequence of the mutual strong interactions, which reduces the zeroth vibrations of the hyperons. This possibility could exist in Universe at temperatures close to $0 \mathrm{~K}$, at the surface of a massive or super-massive black-hole which -in this case, can grow by acquiring also hyper-nuclei, according to the model.

\section{References}

1. M. Arghirescu, "The material structures genesis and field effects", Ed. MatrixROM, Bucharest, (2006)

2. M. Arghirescu, 'The nuclear force explaining by a bag model resulted from a vortexial, cold genesis model of nucleon', Phys. Astron. Int. J. (2018); 2(4):349-358. DOI: 10.15406/paij.2018.02.00109

3. M. Arghirescu, "The Cold Genesis of Matter and Fields", Ed. Science PG, (2015).

4. M. Arghirescu, 'A Quasi-Unitary Pre-Quantum Theory of Particles and Fields and Some Theoretical Implications', IJHEP, July, 80-103, (2015).

5. M. Arghirescu, 'A preonic quasi-crystal quark model based on a cold genesis theory and on the experimentally evidenced neutral boson of 34 me', Global Journal of Physics Vol. 5, No 1, (2016), pp.496-504

6. A. J. Krasznahorkay et al., 'Observation of Anomalous Internal Pair Creation in 8Be: A Possible Signature of a Light, Neutral Boson', arXiv: 1504.01527v1, [nucl-ex], 7 April (2015).

7. R. D. Chipman, L. D. Jennings, Phys. Rev. 132 (1995) 728. CERNCOURIER, 'Precision pins down the electron's magnetism', 4 oct. (2006).

8. ZEUS Collaboration, 'Limits on the effective quark radius from inclusive e-p scattering at HERA' Physics Letters B, 757 (2016) 468-472

9. M. Bhuyan, B. V. Carlson, S. K. Patra, Shan-Gui Zhou, 'Surface properties of neutron-rich exotic nuclei within relativistic mean field formalisms' Phys. Rev. C 97, 024322, 20 Feb. (2018)

10. M. Bhuyan, S. K. Patra, 'Magic Nuclei in Superheavy Valley', Modern Physics Letters A Vol. 27, No. 30, $1250173(2012)$

11. S. K. Biswal, M. Bhuyan et al., 'Search of double shell closure in the superheavy nuclei using a simple effective interaction', Int. Journ. of Modern Physics E, Oct. 2, (2018), pp. 1-15; arXiv:1401.4659v1 [nucl-th] 19 Jan 2014

12. S. Ahmad, M. Bhuyan, S. K. Patra, 'Properties of $Z=120$ nuclei and the $\alpha$-decay chains of the ${ }^{292,304} 120$ isotopes using relativistic and non-relativistic formalism', Int. J. of Mod. Phys. E, Vol. 21, No 11, 1250092 (2012)

13. W. Nazarewicz, 'The limits of nuclear mass and charge', Nature Phys. Vol. 14, (2018), pp. 537-541

14. Yu. Ts. Oganessian, V. K. Utyonkov, 'Super-heavy element research', Reports on Progress in Physics, Vol. 78, No 3, 9 March (2015)

15. W. Bauer, Proc. of 1st Catania Relativistic Ion Studies, Asicastello, Italy (1996) 23

16. T. Lonnroth, Il Nuovo Cimento 110A (1997) 961.

17. T. Schmidt, Z. Phys., 106 (1937) 358.

18. B. Mottelson, Nuclear Structure, Les Houches, Session LXVI, 25 (1996). 
19. J.P. Ebran, E. Khan, T. Niksic', D. Vretenar, 'Cluster-liquid transition in finite saturated fermionic Systems', arXiv:1402.5080v2 [nucl-th] 19 Mars 2014

20. A. Săndulescu et. al - Nucl. Phys., 48 (1963) 345.

21. K. N. Muhin - "Experimental Nuclear Physics", Vol. 1, Ed. "Atomizdat", Moscow (1974).

22. K. A. Gridnev, S. E. Belov, K. V. Ershov, et al., Proc. Int. Conf. on Perspectives in Nucl. Phys., Crete, Greece, Athens (1999).

23. C. F. von Weizsäcker, Z. Phys. 96, p. 431, (1935).

24. K. Kisamori et al., 'Candidate Resonant Tetraneutron State Populated by the $4 \mathrm{He}(8 \mathrm{He}, 8 \mathrm{Be})$ Reaction', Phys. Rev. Lett. 116, 052501, Vol. 116, Iss. 5, (2016)

25. K. Fossez, J. Rotureau, N. Michel, M. Płoszajczak -'Can Tetraneutron be a Narrow Resonance?' Phys. Rev. Lett. 119, 032501 -21 July (2017)

26. Elizabeth A. Donley, N. R., Claussen, S. L. et al. 'Dynamics of collapsing and exploding Bose-Einstein condensates', arXiv: cond-mat/0105019v3, June (2001).

27. W. D. Myers, W. J. Swiatecki, 'Nuclear masses and deformations', Nucl. Phys., Vol. 81, Iss. 1, June (1966), pp. $1-60$

28. G. Royer and C. Gautier, 'On the coefficients and terms of the liquid drop model and mass formula', arXiv:nuclth/0608064v1, (2006).

29. W. Greiner, ,Nuclear Shells in the Superheavy Region within Meson Field Theory', J. Nucl. Radiochem. Sci., Vol. 3, No. 1, (2002), 159-166 